\title{
Quantification of the scratch-healing efficiency for novel zwitterionic polymers
}

\author{
Jan Dahlke ${ }^{1,2}$, Julian Kimmig $\mathbb{B}^{1,2}$, Marcus Abend ${ }^{1,2}$, Stefan Zechel ${ }^{1,2}$, Jürgen Vitz ${ }^{1,2}$, Ulrich S. Schubert $\mathbb{D}^{1,2}$ and \\ Martin D. Hager ${ }^{1,2}$
}

\begin{abstract}
In this work, we present a new strategy to engineer novel self-healing ionomers, namely, zwitterionic polymers, and a comprehensive analysis of their mechanical, viscoelastic, and scratch-healing properties. This new method enables reproducible damage of the polymer surfaces, calculation of the scratch volume through tactile profile scans, and quantification of the self-healing efficiency. Based on the results of the scratch tests and complementary rheology, differential scanning calorimetry (DSC), thermogravimetric analysis (TGA) and hardness tests, new trends, and structure-property relationships can be identified.
\end{abstract}

\section{Introduction}

One of the most important challenges in state-of-the-art research is the development of materials with multiple functions and stimuli responses ${ }^{1,2}$. In modern polymer science, the boundaries of chemistry and material science are becoming blurred. This combination of knowledge is a great opportunity to develop materials with more efficient and sustainable performance. In recent years, a large number of new methods for the synthesis of self-healing synthetics have been developed ${ }^{3-5}$. Polymers are particularly suitable for such approaches due to their versatility in structure and function. Within this context, selfhealing polymers can generally be subdivided into extrinsic and intrinsic healable materials ${ }^{6}$. Extrinsic systems utilize external healing agents, whereas intrinsic selfhealing materials contain functional groups or crosslinks in a network that can be reversibly activated through a stimulus, such as light or heat ${ }^{7}$.

One major advantage of intrinsic approaches is that damage can be healed multiple times in the same area due to the reversible nature of the crosslinks, whereas external

\footnotetext{
Correspondence: Martin D. Hager (martin.hager@uni-jena.de) 'Laboratory of Organic and Macromolecular Chemistry (IOMC), Friedrich Schiller University Jena, Humboldtstraße 10, 07743 Jena, Germany 2Jena Center for Soft Matter (JCSM), Friedrich Schiller University Jena, Philosophenweg 7, 07743 Jena, Germany
}

healing agents are consumed during the curing process ${ }^{8}$. Since the dynamic connections in the network of the polymer form an equilibrium, the cleavage and reconnection can be controlled by external stimuli ${ }^{9}$. In most cases, heat is the trigger for the reaction. This trigger is convenient to apply and further induces mobility in the material, which supports the closing of cavities. However, the equilibrium may also be affected by other environmental conditions, such as (light) irradiation, humidity, $\mathrm{pH}$ value or catalytic agents ${ }^{3}$. Depending on the intended application, an appropriate functional group and reaction type for an intrinsic self-healing polymer is selected, thereby predetermining the nature of the trigger. This chemical toolbox contains various approaches, such as the incorporation of reversible covalent linkers (e.g., DielsAlder (DA) reactions ${ }^{10}$ and imines ${ }^{11}$ ) or supramolecular compounds (e.g., hydrogen bonds ${ }^{12}$ and ionic interactions $)^{13-17}$. For further insights into self-healing polymers with dynamic covalent bonds, interested readers are kindly referred to a recent review ${ }^{3}$. Among the intrinsic self-healing materials with supramolecular interactions, ionomers feature some unique properties compared to other systems ${ }^{18}$. Ionomers can be assigned as a class of polymers with up to $20 \%$ ionic groups in the chemical structure $^{19}$. Usually, acidic groups, e.g., carboxylic acids or phosphates, are integrated as functional groups to serve as 
precursors for ionic moieties ${ }^{20}$. The monomers are readily available or accessible from commercial sources without extensive synthetic procedures, enabling more sustainable and straight-forward production. After modification of the functional units, the ionic groups, which are usually located in the side-chains of the polymer, form aggregates and crosslinks in the polymers. The thermoreversible interactions are also referred to as multiplets or clusters and result in a restricted mobility region around the aggregates $^{21}$. In most cases, this structural change results in a higher glass transition temperature $\left(T_{\mathrm{g}}\right)$ and enhanced mechanical properties compared to the corresponding neutral polymer ${ }^{22}$. The Eisenberg-Hird-Moore model described this theoretical concept in the early $1990 \mathrm{~s}^{23,24}$. The dynamic nature of the physical crosslinking in ionomers through the ionic groups allows processing the polymer (at elevated temperatures) while maintaining toughness and mechanical properties. Thus, one can classify these materials as thermoplastic elastomers ${ }^{19}$.

The commercial potential for ionomers was recognized in the 1960 s by DuPont, who commercialized polyethylene containing low amounts of functional groups with zinc or sodium carboxylates under the name of Surlyn ${ }^{\circledR}$, which is currently the most studied ionomer for self-healing applications ${ }^{25,26}$. Neutralized poly(ethyleneco-methacrylic acid) is able to heal damage after ballistic puncture $^{27}$. The high energy of the projectile dissipates heat around the edges of the damaged site, which triggers an elastic response of the surrounding material, increases the mobility and enables the material to flow and close the cavity. The mechanical integrity is restored through a rearrangement of the ionic clusters ${ }^{28}$. Varley et al. demonstrated the influence of the ionic content on the healing and physical properties of the polymer ${ }^{25}$. Generally, a high content of ionic groups leads to increased plastic behavior and less elasticity. Lower mobility is disadvantageous at lower temperatures, whereas lower mobility benefits the viscoelastic properties at elevated temperatures and in the melt state ${ }^{25}$.

In addition to the described studies of polyethylenebased ionomers, only a few other ionomers with highmechanical stability have been investigated in terms of their self-healing ability ${ }^{29-33}$. For example, Bose et al. ${ }^{34,35}$ performed extensive rheological studies for poly(butyl acrylate)-based ionomers and found correlations between their network dynamics and self-healing behavior. In our previous work, we demonstrated the healing capabilities of ionomers containing carboxylate and phosphate groups in different polymeric systems ${ }^{36}$.

In this study, we present the synthesis and detailed investigation of zwitterionic polymer networks with selfhealing behavior to gain a deeper understanding of the healing, mechanical and chemical properties of these supramolecular self-healing polymers. The investigation includes an assessment of the viscoelastic properties, the mechanical hardness and a new reproducible and quantifiable approach towards sophisticated scratch-healing tests that can be extended and applied to many other materials. The new method allows imaging of scratches through tactile profile scans and subsequent calculations of a three-dimensional (3D) image of the surface and the scratch volume under the surface. This method is a first step towards the investigation of "real-life" scratches, i.e., scratches that show a more complex damage pattern (e.g., fish-scale pattern). The presented results describe a comprehensive analysis of the structure-property relationships of zwitterionic polymers with particular attention to the surface healing behavior.

Polyzwitterions, also known as polybetaines, contain positive and negative charges in the functional groups of the polymer. The ionic groups are usually distributed along the main chain or in the side-chains of the polymer $^{37}$. Polyzwitterions are a class of materials with a multitude of possible structures and synthetic approa$\mathrm{ches}^{38}$. The synthesis of water-based reactions and hydrogels with a high-ionic content is conventionally realized by polymerization of the ionic monomers ${ }^{39,40}$. However, the combination of hydrophobic comonomers can be difficult in view of the solvent and the reactivity of the polymerizable units. In these cases, a neutral copolymer can be synthesized and subsequently functionalized by addition reactions to introduce the ionic groups ${ }^{41,42}$. Although the materials often have hydrophilic characteristics due to their strong Coulomb interactions, in particular with high-ionic contents, polyzwitterions otherwise behave more like nonionic polymers rather than conventional polyelectrolytes ${ }^{38,43}$. One of the most common applications is zwitterionic hydrogels, which can be responsive to environmental conditions, such as the $\mathrm{pH}$ value, temperature or salt content ${ }^{44-46}$. It is also possible to combine different effects in one material, such as shape-memory and moisture sensitivity or even selfhealing ${ }^{44,47,48}$. The corresponding applications range from biomedical materials and antifouling surfaces to dispersants and stabilizers ${ }^{37,49-51}$.

\section{Materials and methods}

A detailed description of the synthesis and characterization of all materials can be found in the Supporting Information.

\section{Results and discussion}

In this work, a series of zwitterionic copolymers containing sulfobetaine moieties as functional groups is prepared and characterized. The ionomers contain varying contents of ionic groups between 5 and $17 \%$ to study the effect of increasing amounts of zwitterionic 
structures and crosslinks in the network. Furthermore, two different spacer lengths were introduced between the heteroatoms carrying the charges in the side-chains. Three ionic polymers with a shorter C3-spacer were synthesized containing 3-((2-(methacryloyloxy)ethyl) dimethylammonium) propane sulfonate (MAPS); moreover, 3-((2-(methacryloyloxy)ethyl) dimethylammonium) butane sulfonate (MABS) was utilized to separate the heteroatoms with a $\mathrm{C} 4$ spacer in three additional ionic copolymers. An overview of the synthetic route is given in Schemes 1 and 2.

For the synthesis of the zwitterionic polymers, a polymerization strategy based on butyl methacrylate (BMA) and dimethylamino ethyl methacrylate (DMAEMA) was chosen. Thus, it is possible to synthesize two types of zwitterionic copolymers from one batch of neutral polymer precursors, which enables good comparability because the molar mass and the dispersity are as close as possible.

\section{Synthesis and characterization of the copolymers P1 through P3}

The synthesis of the neutral copolymers P1 through P3 was achieved by reversible addition-fragmentation chain

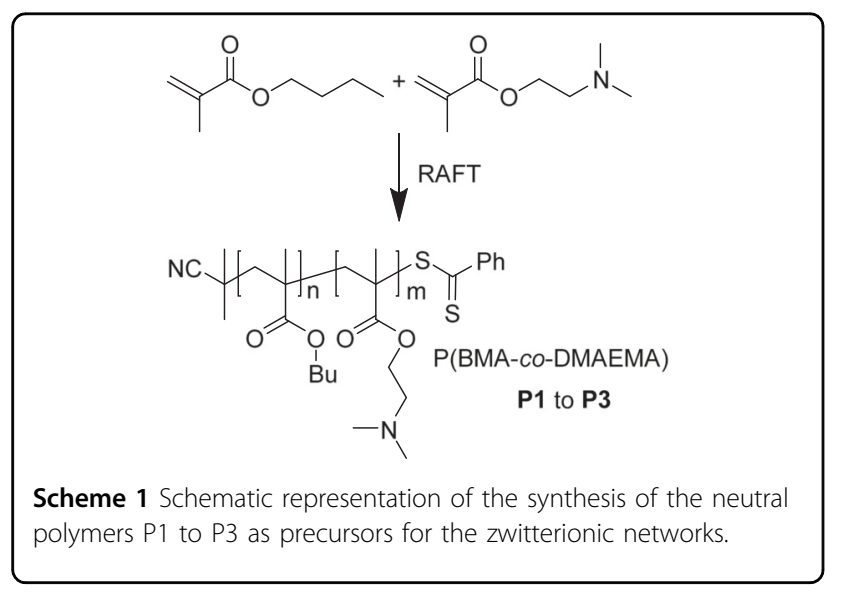

transfer (RAFT) polymerization of BMA and DMAEMA in dimethylformamide (DMF) with AIBN as the initiator. The RAFT method was utilized to perform a controlled polymerization process resulting in a low dispersity and controlled molar masses ${ }^{52}$. The detailed reaction procedures are given in the Supporting Information (SI). After purification via dialysis in acetone, the resulting copolymers were characterized by nuclear magnetic resonance (NMR)-spectroscopy and size exclusion chromatography (SEC). The ratio of the monomers in the polymers was calculated from the NMR signals of the corresponding $\mathrm{OCH}_{2}$ and $\mathrm{NCH}_{2}$ signals, respectively (see Table 1 ). The results are in good agreement with the monomer ratios utilized in the polymerization reactions. A comparison of the molar masses of the copolymers indicates a decreasing molar mass with increasing DMAEMA content while maintaining the same reaction conditions, such as initiator ratio and chain transfer agent content. The molar masses $\left(M_{\mathrm{n}}\right)$ ranged from $11,800 \mathrm{~g} / \mathrm{mol}$ for $\mathbf{P 3}$ to $16,000 \mathrm{~g} / \mathrm{mol}$ for P1 (see Table 1).

\section{Synthesis and characterization of the zwitterionic copolymers Z1 through Z6}

To prepare the zwitterionic copolymers, a straightforward synthesis route starting from P1 through P3 was chosen (for a detailed description, see the SI). The content of the ionic groups was calculated from the NMR ratios, as shown in Table 1 .

The polymers were stirred for four days with the sultone in tetrahydrofurane (THF) until completion of the reaction (according to NMR). The ${ }^{1} \mathrm{H}$ NMR signals of DMAEMA in the starting materials $\left(\mathrm{NCH}_{2}\right.$ and $\left.\mathrm{N}\left(\mathrm{CH}_{3}\right)_{2}\right)$ were utilized as an indicator for the reaction conversion since the signals shift downfield after quaternization (from $2.6 \mathrm{ppm}$ to $3.3 \mathrm{ppm}$ ). The reaction with 1,4-butanesultone (Z4 through Z6) requires higher temperatures compared to the synthesis of MAPS ( $\mathbf{Z 1}$ through $\mathbf{Z 3}$ ) due to the lower reactivity. As a result of the large batch reactions that were required for further characterization, all products were purified through dialysis.

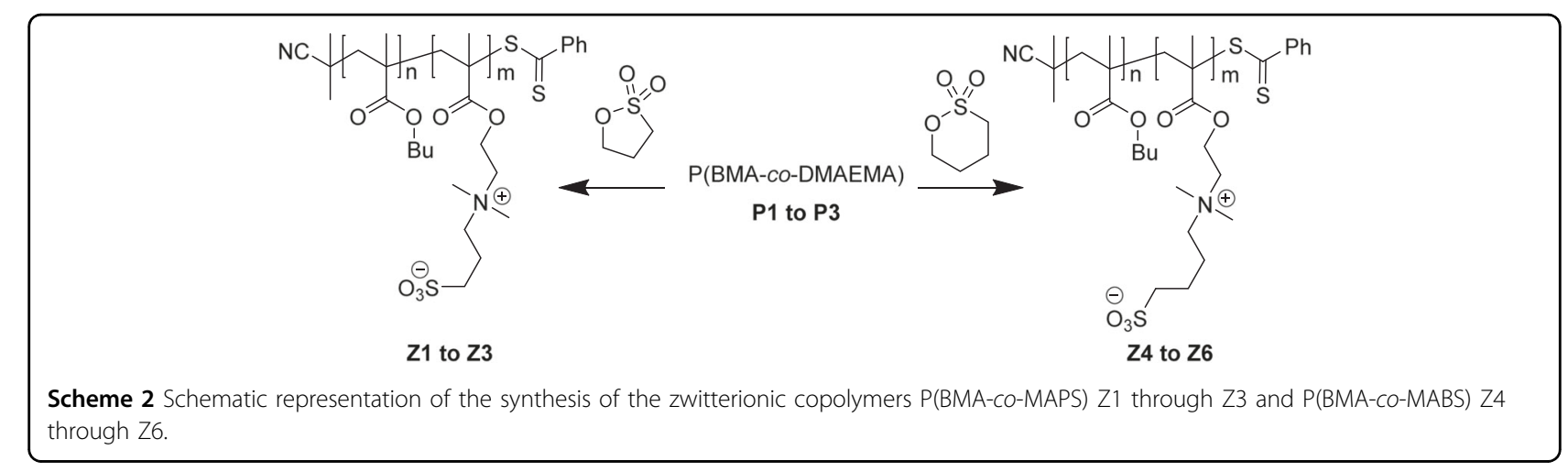


Table 1 Calculation of the monomer ratios for the copolymers P1 to P3 from NMR and the molar masses from SEC (THF, PMMA standard).

\begin{tabular}{llllll}
\hline Polymer & $\begin{array}{l}\text { Ratio (BMA/ } \\
\text { DMAEMA) }\end{array}$ & $\begin{array}{l}\text { Content } \\
\text { DMAEMA [\%] }\end{array}$ & $\begin{array}{l}\boldsymbol{M}_{\mathbf{n}}[\mathbf{g} / \\
\mathbf{m o l}]\end{array}$ & $\begin{array}{l}\boldsymbol{M}_{\mathbf{w}}[\mathbf{g} / \\
\mathbf{m o l}]\end{array}$ & $\boldsymbol{\theta}$ \\
\hline P1 $(\mathrm{Z} 1, \mathrm{Z} 4)^{\mathrm{a}}$ & 17.3 & 5.5 & 16,000 & 20,600 & 1.29 \\
P2 $(\mathrm{Z} 2, \mathrm{Z} 5)^{\mathrm{a}}$ & 8.4 & 10.6 & 13,100 & 17,700 & 1.35 \\
P3 $(\mathrm{Z3}, \mathrm{Z6})^{\mathrm{a}}$ & 4.8 & 17.2 & 11,800 & 16,100 & 1.37 \\
\hline
\end{tabular}

${ }^{a}$ The corresponding ionomers $(Z)$, which were prepared from the neutral polymers $(P)$ are given in the round parenthesis. According to the NMR spectra of the polyzwitterions, full conversion was reached for the functionalization reactions and, thus, the ionic content of the ionomers Z1 to Z6 is equal to the monomer ratios found for the corresponding neutral polymers in this table

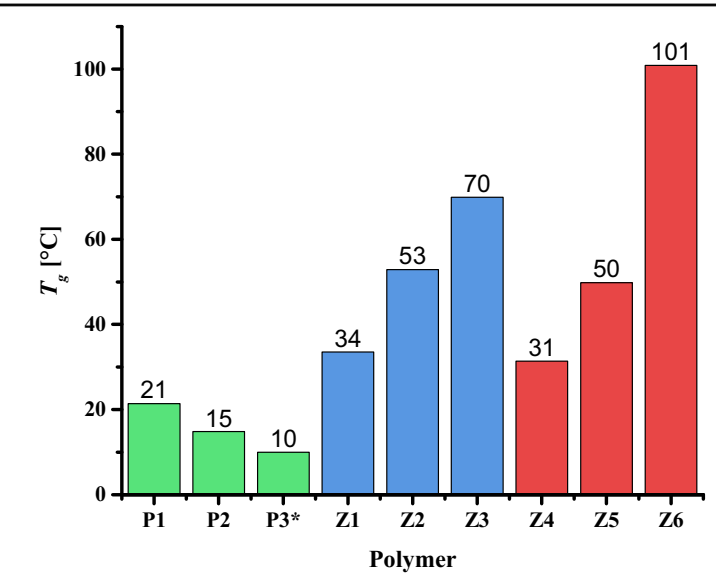

Fig. 1 Summary of the glass transition temperatures for all copolymers and zwitterionic copolymers. The $T_{\mathrm{g}}$ for $\mathbf{P} \mathbf{3}$ is

estimated; the $T_{\mathrm{g}}$ could not be calculated directly from the DSC curve (see the SI)

\section{Differential scanning calorimetry (DSC) and thermogravimetric analysis (TGA)}

For the designated application as self-healing materials, a detailed investigation of the thermal properties of the polymers is essential. Thus, the thermal stability was analyzed by TGA. The results showed that all neutral copolymers and zwitterionic copolymers are temperature stable up to $200^{\circ} \mathrm{C}$ and higher. The exact values and TGA curves for the decomposition temperatures $\left(T_{\mathrm{d}}\right)$ are given in the SI. A healing and processing temperature of up to $150^{\circ} \mathrm{C}$ is well suited for this type of polymer. As described earlier, the content of ionic groups and the corresponding crosslinking density in the polymer network has an influence on the thermal behavior and the glass transition temperature $\left(T_{\mathrm{g}}\right)$. For the healing process to occur in a polymer, a certain physical flow of the material is required. The glass transition can be utilized to increase the mobility in the polymer because temperature is a suitable trigger for the reversible ionic interactions in self-healing ionomers. Therefore, DSC measurements were performed to evaluate the effect of the chemical structure of the polymer on the physical properties and to further estimate the environmental conditions, which are required to process and heal the polymers. A summary of the results of the DSC measurements is depicted in Fig. 1. Additionally, all curves can be found in the SI. The copolymers and ionomers show large differences in their $T_{\mathrm{g}}$-values, and some trends can be elucidated. First, the neutral copolymers show a decreasing $T_{\mathrm{g}}$ with increasing DMAEMA content. P1, which has the lowest comonomer content, features a $T_{\mathrm{g}}$ close to the theoretical value of the PBMA homopolymer, whereas samples P2 and P3 have a lower $T_{\mathrm{g}}{ }^{53}$. In this case, the functional moiety can be considered as a softener in the material. However, with the ionomers, a higher content of ionic groups leads to more crosslinks through ionic interactions in the network and to a higher $T_{\mathrm{g}}$-value, which was also observed for other polymethacrylates containing zwitterionic comonomers ${ }^{54}$. For the highest content of the comonomers, the MABS-containing copolymers exhibited the highest $T_{\mathrm{g}}$-value due to the higher dipole moment of the monomer with the butyl spacer compared to that with the propyl spacer ${ }^{55,56}$. No melting point was found for any of the samples described.

\section{Vickers hardness}

One of the benefits of ionomeric polymer systems is their good mechanical performance and stability at room temperature. The Vickers hardness test represents a very potent measurement method to evaluate the mechanical resistance of polymer surfaces ${ }^{57}$. For this measurement, a special square-based pyramidal diamond indenter is utilized, which exerts a certain amount of normal force (F) on the surface of the polymers over a certain amount of time; in this case, the force was $0.1 \mathrm{~N}$ with a holding time of $30 \mathrm{~s}$. Subsequently, the residual imprint on the surface was analyzed with a microscope to determine the average length of the diagonal of the square (d). The Vickers hardness number (HV) can be calculated with the following formula $(k=$ constant depending on the indenter geometry $)^{58}$ :

$$
\mathrm{HV}=\frac{F}{A}=k \frac{F}{d^{2}}
$$

The samples were tested ten times each, and the average Vickers hardness was utilized hereinafter for conclusions. A detailed description of the measurement can be found in the SI, and a summary is depicted in Fig. 2. Consistent with the DSC results, one can identify similar trends for the hardness of the polymers and ionomers. The neutral copolymers P1 through P3 exhibited the lowest hardness between 2.2 and 1.4 with decreasing hardness at higher DMAEMA-monomer contents; this trend was also 


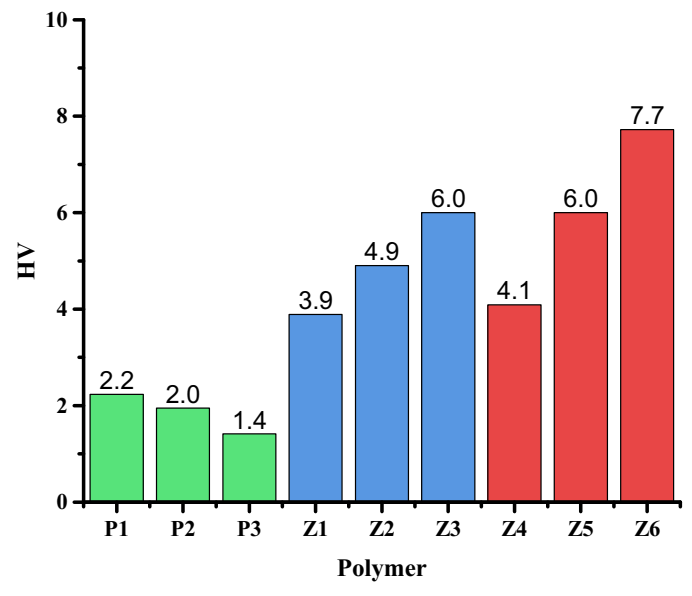

Fig. 2 Summary of the Vickers hardness measurements (HV 0.1/30) for all copolymers and zwitterionic copolymers.

observed for the $T_{\mathrm{g}}$-value. Significantly higher hardness values can be found for the ionomers. As expected, the increasing ionic nature of the network leads to higher hardness for both types of zwitterionic polymers. Furthermore, samples $\mathbf{Z 4}$ through $\mathbf{Z 6}$ containing MABS as a functional unit exhibited a slightly higher hardness than the corresponding zwitterionic copolymer $\mathbf{Z 1}$ through Z3 with the same content of MAPS, which is presumably due to the higher dipole moment of the MABS (see the thermal properties). Thus, the mechanical performance of the material can be conveniently adjusted by the number of functional moieties introduced into the polymer structure.

\section{Rheology}

The viscoelastic properties of the polymers and polyzwitterions were determined by rheological investigations and dynamic mechanical analysis (DMA). For this purpose, test specimens of each polymer were fabricated. Such specimens are conventionally prepared by direct polymerization of a monomer bulk mixture in the designated mold. However, in this particular case, this method is not applicable due to the solution polymerization and the postmodification of the polymers. Furthermore, we wanted to ensure good comparability by utilizing the same batch of polymer for each specimen. An advantage of ionomers compared to other crosslinked polymers is the good processability at elevated temperatures ${ }^{18}$. Consequently, a tailor-made hot press mold was utilized to prepare specimens with a size of $30 \times 10 \mathrm{~mm}$ and a thickness of 2 to $4 \mathrm{~mm}$. The polymers were filled in the mold and annealed at the required temperature (between 80 and $\left.130^{\circ} \mathrm{C}\right)$. Subsequently, the polymers were hotpressed with a load of $1.5 \mathrm{t}$ for $5 \mathrm{~min}$ to ensure a bubbleand defect-free specimen. A solid rectangular fixture system (SRF) was utilized in the DMA measurements. The details of the measurements and the resulting curves are described and depicted in the SI. The polymers P1 through P3 show typical behavior of amorphous noncrosslinked polymers. At room temperature, the storage modulus $\left(G^{\prime}\right)$ is approximately one magnitude higher than the loss modulus $\left(G^{\prime \prime}\right)$, which indicates mainly elastic behavior. A crossover of $G^{\prime}$ and $G^{\prime \prime}$ between 36 and $53^{\circ} \mathrm{C}$ indicates a change in the viscoelastic behavior for the neutral polymers towards increasing viscous behavior. These results are in good agreement with the observations made during hot pressing. At $\sim 80^{\circ} \mathrm{C}$, the polymers are already soft and can be pressed into the desired shape. Complementing the findings, the complex viscosity $\left(\eta^{*}\right)$ in the same area decreases. The decrease in $G^{\prime}$ is less prominent and steep for the zwitterionic copolymers $\mathbf{Z 1}$ through Z3. The polyzwitterions with the longer spacer in the side-chains ( $\mathbf{Z 4}$ to $\mathbf{Z 6}$ ) have a notably larger gap between $G^{\prime}$ and $G^{\prime \prime}$ at low temperatures. This finding indicates an even higher elastic share of the viscoelastic behavior. As expected, the area of elastic behavior (before the intersection of the curves of $\mathrm{G}^{\prime}$ and $\mathrm{G}^{\prime \prime}$ ) is larger for all ionomers compared to that of the corresponding neutral polymers. The crossover of the neutral polymers is below $52^{\circ} \mathrm{C}$, whereas the ionomers show intersection points over $96^{\circ} \mathrm{C}$, in which the highest values are found for ionomers $\mathbf{Z 4}$ through $\mathbf{Z 6}$ (see Fig. 3); these findings correlate to the thermal properties and the hardness values.

The deformation behavior of polymers at a certain temperature is of great importance for further rheological investigations and is characterized by the nondestructive deformation limit, which is also referred to as the linear viscoelastic (LVE) region. In this area, the sample can be deformed without permanent destruction of the mechanical integrity. The LVE region is determined by amplitude tests, in which the shear deformation is increased at constant temperature and frequency. For this purpose, the polymers P1, P2, Z1, and Z5 were measured at room temperature and at $50{ }^{\circ} \mathrm{C}$. The LVE region is defined by the area of constant $\mathrm{G}^{\prime}$ and $\mathrm{G}^{\prime \prime}$ until the first drop. The neutral copolymer $\mathbf{P 1}$ is stable until a deformation of $0.1 \%$ at $25^{\circ} \mathrm{C}$. All other tested samples (P2, Z1, and $\mathbf{Z 5}$ ) show an LVE region up to 0.3 to $0.5 \%$ at room temperature. The first drop indicates mechanical damage due to the deformation in the polymer matrix. A steep drop in G' generally indicates brittle fracture behavior, which can be observed for all samples tested at room temperature and is consistent with the observations from the processing of the polymers and ionomers. However, at $50{ }^{\circ} \mathrm{C}$, the LVE region extends for all measurements to $\gamma$ of $1 \%$, and the samples show less brittleness. Therefore, hereinafter, it is possible to carry out frequency sweep tests at $\gamma=0.1 \%$ without concern for fracture of the specimen. 


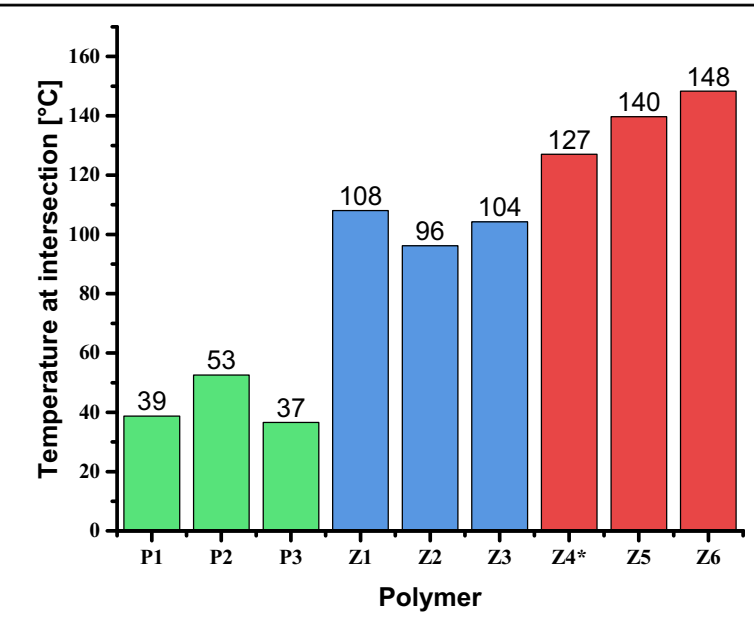

Fig. 3 Summary of the crossover points of $G^{\prime}$ and $G^{\prime \prime}$ of the temperature-dependent rheology studies ( ${ }^{*}$ two additional intersection points were found for the ionomer $Z 4$ at 69.2 and $54.2^{\circ} \mathrm{C}$, which were not considered for this graphical representation, as shown in the SI).

Based on the results of the temperature sweeps, the frequency sweeps were performed at $100^{\circ} \mathrm{C}$. Supramolecular polymer networks usually show a crossover of $G^{\prime}$ and $G^{\prime \prime}$ in the frequency sweep ${ }^{34,59,60}$. The crossover is related to the dynamic nature of the interactions in the material. In agreement with the considerations, a crossover at $\sim 1 \mathrm{~Hz}$ was found for the zwitterionic copolymers $\mathbf{Z 1}$ through $\mathbf{Z 4}$. At low frequencies, the viscous share of the viscoelastic behavior prevails, whereas after the crossover, the storage modulus dominates. For a polymer with permanent crosslinking, the storage modulus $G^{\prime}$ would dominate $G^{\prime \prime}$ over the whole range of frequencies. This behavior can be found for $\mathbf{Z 5}$ and $\mathbf{Z 6}$ at $100{ }^{\circ} \mathrm{C}$ because the polymers are rigid and the intersection of $G^{\prime}$ and $G^{\prime \prime}$ in the temperature sweeps is much higher at 139 and $148{ }^{\circ} \mathrm{C}$, respectively. The results complement the findings from the self-healing study in the following section because $\mathbf{Z 5}$ and $\mathbf{Z 6}$ show the lowest healing efficiencies. In addition, the overall storage modulus of the ionomers increases with increasing ionic groups and crosslinking density.

\section{Self-healing studies}

The quantitative evaluation of the self-healing ability of polymers has been a challenge since the beginning of research on healable materials. Thus, several definitions of healing efficiency are common depending on the damage type, the utilized trigger, the external conditions and the healing process itself $^{61,62}$.

Mechanically tough polymers, which are able to heal damage, are particularly suitable for coatings and adjacent applications. Thus, scratch-healing tests are naturally very important studies for such materials. In most cases, the evaluation of the healing is performed by optical analysis of the microscopy images or calculation of the damaged surface area before and after the healing process ${ }^{63}$.

However, the quantification of the healing efficiency is much more complex. Ideally, the volume of a scratch rather than the surface area should be calculated. In many cases, the analysis and calculation of scratch volumes by optical microscopy is not feasible due to the limited reflection of the light from the inner walls of the scratches. Very small defects on flat surfaces can be investigated by atomic force microscopy ${ }^{64,65}$. In this work, we present a new method for the controlled and reproducible introduction of scratches on surfaces and the imaging and quantification of the same scratches through tactile measurements. In this manner, more complex damage behavior can also be studied, which can also be a result of "reallife" damage, e.g., fish-scale pattern formation (a pattern, which is common in scratch maps of polymers) ${ }^{66,67}$.

For this purpose, hot-pressed ionomer specimens were embedded in an epoxy resin matrix before the surface was ground and polished. A detailed description of the process is given in the SI. The samples were scratched over a length of $2500 \mu \mathrm{m}$ with a diamond indenter and imaged utilizing an $\mathrm{MST}^{3}$ scratch tester from Anton Paar. Subsequently, the samples were turned $90^{\circ}$, and the surface profile was measured with a low-contact force of $5 \mathrm{mN}$ along the full length of the scratch (160 profiles every $20 \mu \mathrm{m})$. The data set was then compiled into a 3D image of the surface, and the volume below the original surface was calculated after baseline correction utilizing the undamaged surrounding surface. This measurement was repeated after each healing step. The resulting volumes of the initial scratched surface $\left(V_{\mathrm{I}}\right)$ and the healed surface $\left(V_{\mathrm{H}}\right)$ were the basis for calculating the healing efficiencies.

$$
H_{\text {eff }}=100 \%\left(1-\frac{V_{\mathrm{H}}}{V_{\mathrm{I}}}\right)
$$

All ionomers $\mathbf{Z 1}$ through $\mathbf{Z 6}$ were scratched and imaged by the same program to allow good comparability of the healing behavior (see Table 2). The detailed parameters can be found in the SI. After scratching the surfaces, the samples were healed at $120^{\circ} \mathrm{C}$ for $2 \mathrm{~h}$ and then for an additional $4 \mathrm{~h}$. The volume of the residual scratch was calculated after every healing step. Additional healing experiments at $55^{\circ} \mathrm{C}$ for the neutral copolymers P1 through P3 can be found in the SI. The temperature was chosen according to the results of the crossover of $\mathrm{G}^{\prime}$ and G" in the temperature sweeps. The neutral polymers exhibited much worse healing performance than the zwitterionic structures. However, it should be noted that a fair comparison between these neutral polymers and the supramolecular ionomer networks is difficult because the mechanical and thermal properties are very different. 
Table 2 Results of the self-healing experiments with the calculated healing efficiency for the zwitterionic polymers Z1 to Z6.

\begin{tabular}{|c|c|c|c|c|}
\hline Ionomer & $\begin{array}{l}\text { Functional } \\
\text { group }\end{array}$ & $\begin{array}{l}\text { Content of ionic } \\
\text { group (\%) }\end{array}$ & $\begin{array}{l}H_{\text {eff }} \\
2 \mathrm{~h} 120^{\circ} \mathrm{C}(\%)\end{array}$ & $\begin{array}{l}H_{\text {eff }} \\
6 \mathrm{~h} 120^{\circ} \mathrm{C}(\%)\end{array}$ \\
\hline Z1 & MAPS & 5.5 & 63 & 99 \\
\hline Z2 & MAPS & 10.6 & 77 & 80 \\
\hline Z3 & MAPS & 17.2 & 98 & 98 \\
\hline Z4 & MABS & 5.5 & 24 & 89 \\
\hline Z5 & MABS & 10.6 & 18 & 49 \\
\hline Z6 & MABS & 17.2 & 13 & 35 \\
\hline
\end{tabular}

The healing tests revealed significant differences between the tested zwitterionic polymers. After the first $2 \mathrm{~h}$ at $120^{\circ} \mathrm{C}$, the ionomers $\mathbf{Z 1}$ through $\mathbf{Z 3}$ (with the short spacer (MAPS) between the positive and the negative charge) showed a healing efficiency above $60 \%$. In this case, a higher ionic content led to increased healing efficiency up to nearly complete healing for Z3 (98\%). After a total healing time of $6 \mathrm{~h}$ at $120^{\circ} \mathrm{C}$, all three samples could increase the efficiency even further. $\mathbf{Z 2}$ exhibited the lowest healing ability of the three at $80 \%$. Optical images of a scratch and the healed surface and the 3D-plotted surface for $\mathbf{Z 1}$ are depicted in Figs. 4 and 5, respectively.

However, the corresponding ionomers with the longer

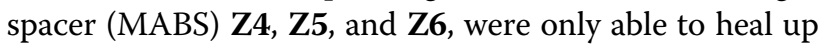
to $24 \%$ of the scratch after $2 \mathrm{~h}$. In contrast to the other MAPS-based ionomers, these materials show a decreased healing efficiency with more ionic groups within the structure, which also correlates with the previously discussed thermal and mechanical properties. The polyzwitterion $\mathbf{Z 6}$ healed $13 \%$ after $2 \mathrm{~h}$ at the tested healing conditions. After four additional hours at $120^{\circ} \mathrm{C}$, only $\mathbf{Z 4}$ was able to heal the damage with an efficiency up to $89 \%$. The samples $\mathbf{Z 5}$ and $\mathbf{Z 6}$, which contain a much higher content of crosslinks within their structure than $\mathbf{Z 4}$, did not exceed an efficiency of 49\% (Z5) and 35\% (Z6). Based on the theoretical concept of ionomers, the higher ionic group content decreases the mobility within the material due to the extended areas of physical crosslinks, thereby limiting the elastic responsiveness of the polymers even at elevated temperatures ${ }^{24}$. The results are complemented by the frequency sweeps of rheological measurements, as discussed earlier. For these types of polymers, a higher healing temperature is expected to be beneficial. However, within the scope of this work, $120^{\circ} \mathrm{C}$ was found to be the optimal healing temperature to compare the synthesized ionomers and to identify structural property dependencies. On the basis of the results, a clear influence of the chemical structure on the mechanical properties and the healing behavior was determined. The incorporation of zwitterionic side-chains increased the mechanical stability and the $T_{\mathrm{g}}$-value in comparison to the corresponding

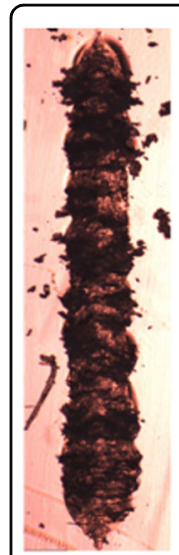

Scratch

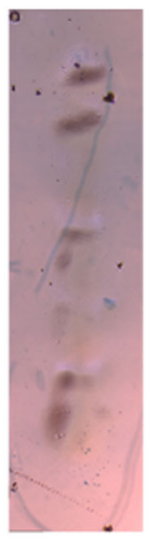

$2 \mathrm{~h}, 120^{\circ} \mathrm{C}$

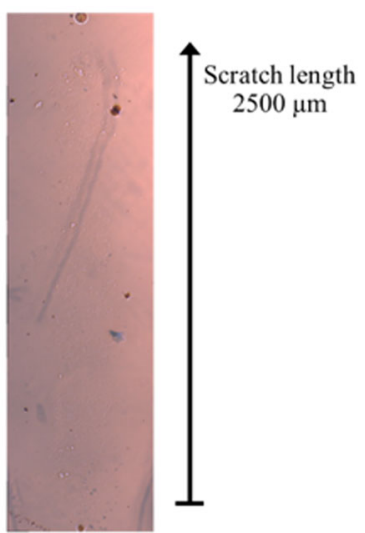

$6 \mathrm{~h}, 120^{\circ} \mathrm{C}$
Fig. 4 Optical images of the scratch on the surface and the healed scratch after $2 \mathrm{~h}$ and $6 \mathrm{~h}$ at $120^{\circ} \mathrm{C}$ (left to right) for ionomer $\mathrm{Z1}$.

neutral polymers due to supramolecular crosslinking. Furthermore, the ionic group content was responsible for the rigidity in the material. A higher content was favorable for the mechanical stability of the polymer network. Even more interesting were the differences between the two tested functional moieties MAPS and MABS. The chain extension by one $\mathrm{CH}_{2}$ unit led to different mechanical, thermal and viscoelastic properties. The longer C4-linker increased the $T_{\mathrm{g}}$-values and the Vickers hardness compared to the shorter C3-linker. However, the higher rigidity apparently was unfavorable for healing efficiency. The ionomers $\mathbf{Z 4}, \mathbf{Z 5}$, and $\mathbf{Z 6}$ all exhibited worse healing behavior than $\mathbf{Z 1}$ through $\mathbf{Z 3}$ in relation to the performed scratch-healing tests. However, the healing efficiency was always dependent on the healing conditions. The investigated structure-property relations can be utilized to design tailor-made zwitterionic copolymers for selfhealing applications at different temperatures and in varying timeframes.

\section{Conclusions}

The series of new ionomers presented in this work contain two different functional zwitterionic side groups (MAPS and MABS) with varying content. The straightforward synthesis enabled a good comparability of the six ionomers and the identification of structure-property relationships. The thermal properties showed that the $T_{\mathrm{g}}$-value was clearly dependent on the functional group content in the polymer networks. Moreover, the Vickers hardness behaves in a similar way and supports the results. The materials were further investigated by rheological studies to determine the viscoelastic behavior. The elastic share of this behavior at the tested temperatures increased with higher ionic content and longer chain length of the spacer. This behavior had a direct influence 

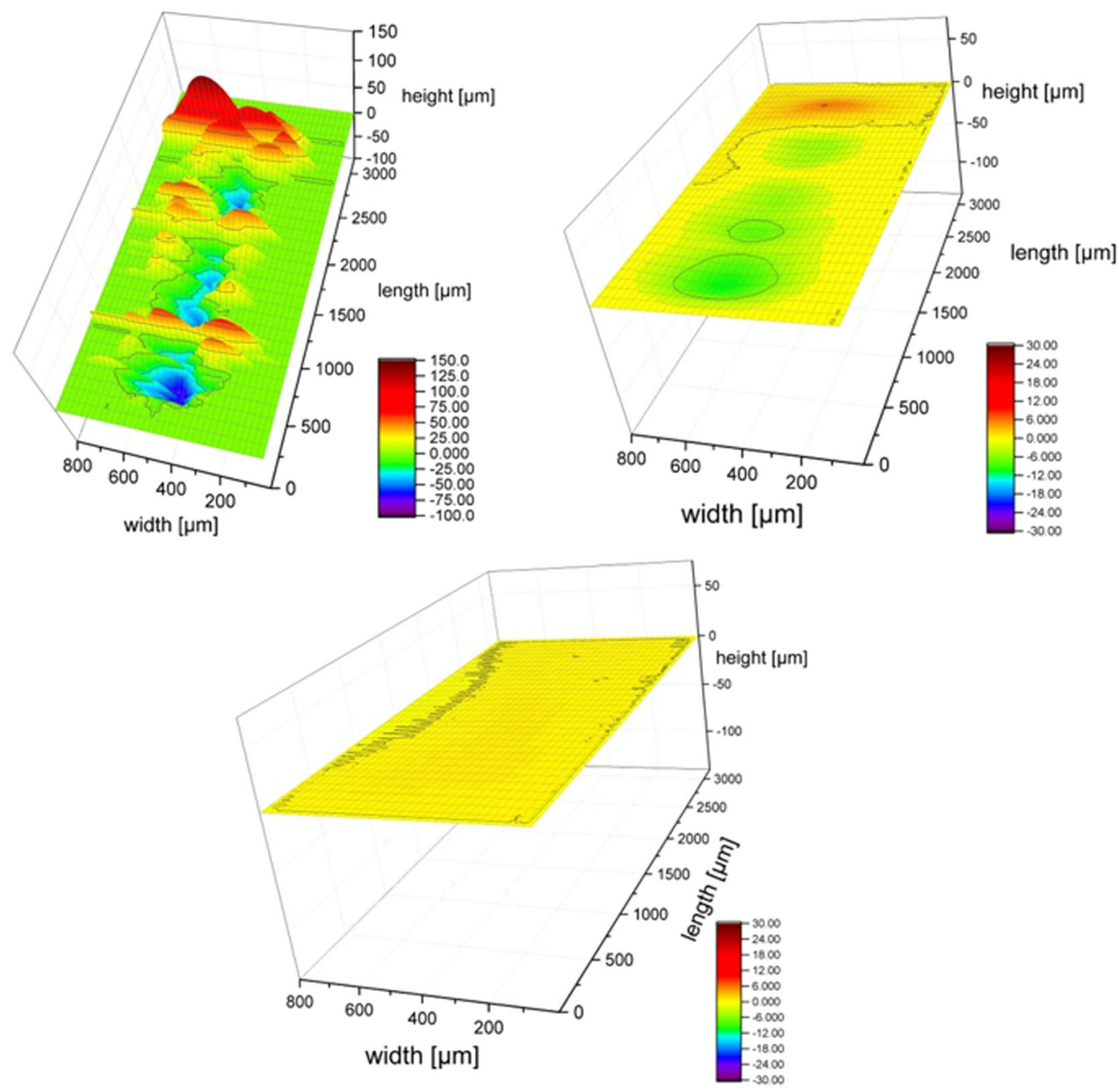

Fig. 5 Top view of the 3D-plotted scratch from the profile measurements before healing (top left), after $2 \mathrm{~h}$ at $120^{\circ} \mathrm{C}$ (top right) and after $6 \mathrm{~h}$ at $120^{\circ} \mathrm{C}$ (bottom) for ionomer Z1.

on the self-healing properties and enabled the preparation of tailor-made ionomers for predetermined healing conditions and applications. To establish a quantifiable and reliable scratch-healing evaluation method, the polymer specimens were hot-pressed, embedded into epoxy resin and scratched with a Rockwell indenter on a microscratch tester. Subsequently, the damaged surfaces were measured with tactile profile scans, which enabled the investigation of more complex patterns and the calculation of the scratch volume. The process was repeated after the healing process, through which a healing efficiency could be calculated. The ionomers $\mathbf{Z 1}$ through $\mathbf{Z 3}$ containing the MAPS group exhibited better healing behavior at $120^{\circ} \mathrm{C}$ than the MABS-containing polymer networks. For the latter, a clear influence of the ionic content was identified. The established scratch test method offers a new possibility to determine trends and tendencies for self-healing polymers in relation to their structure and is in good agreement with the investigation of the mechanical and viscoelastic properties. This method can be extended to other self-healing materials for surface applications and allows reproducible scratching and healing efficiency quantification.

\section{Acknowledgements}

We gratefully acknowledge the Deutsche Forschungsgemeinschaft (DFG) for financial support (SPP 1568 and HA606/7-1). S.Z. is grateful to the Carl-Zeiss foundation for funding.

Conflict of interest

The authors declare that they have no conflict of interest.

\section{Publisher's note}

Springer Nature remains neutral with regard to jurisdictional claims in published maps and institutional affiliations. 
Supplementary information is available for this paper at https://doi.org/ 10.1038/s41427-019-0190-2.

Received: 23 June 2019 Revised: 28 August 2019 Accepted: 17 September 2019.

Published online: 17 January 2020

\section{References}

1. Wei, M., Gao, Y., Li, X. \& Serpe, M. J. Stimuli-responsive polymers and their applications. Polym. Chem. 8, 127-143 (2017).

2. Stuart, M. A. C. et al. Emerging applications of stimuli-responsive polymer materials. Nat. Mater. 9, 101-113 (2010).

3. Dahlke, J., Zechel, S., Hager, M. D. \& Schubert, U. S. How to design a selfhealing polymer: general concepts of dynamic covalent bonds and their application for intrinsic healable materials. Adv. Mater. Interfaces 5, 1800051 (2018).

4. van Dijk, N. \& van der Zwaag, S. Self-healing phenomena in metals. Adv. Mater. Interfaces 5, 1800226 (2018).

5. Yang, Y. \& Urban, M. W. Self-healing of polymers via supramolecular chemistry. Adv. Mater. Interfaces 5, 1800384 (2018).

6. Hager, M. D, Zwaag, van der, S. \& Schubert, U. S. Self-Healing Materials. (Springer, Switzerland, 2016).

7. Blaiszik, B. J. et al. Self-healing polymers and composites. Annu. Rev. Mater. Res. 40, 179-211 (2010).

8. Garcia, S. J. Effect of polymer architecture on the intrinsic self-healing character of polymers. Eur. Polym. J. 53, 118-125 (2014).

9. Roy, N., Bruchmann, B. \& Lehn, J. -M. DYNAMERS: dynamic polymers as selfhealing materials. Chem. Soc. Rev. 44, 3786-3807 (2015).

10. Chen, $X$. et al. A thermally re-mendable cross-linked polymeric material. Science 295, 1698-1702 (2002).

11. Li, H., Bai, J., Shi, Z. \& Yin, J. Environmental friendly polymers based on schiffbase reaction with self-healing, remolding and degradable ability. Polymer $\mathbf{8 5}$ 106-113 (2016).

12. Cordier, P., Tournilhac, F., Soulié-Ziakovic, C. \& Leibler, L. Self-healing and thermoreversible rubber from supramolecular assembly. Nature 451, 977-980 (2008).

13. Varley, R. J. \& van der Zwaag, S. Towards an understanding of thermally activated self-healing of an ionomer system during ballistic penetration. Acta Mater. 56, 5737-5750 (2008).

14. Scheltjens, G., Diaz, M. M., Brancart, J., van Assche, G. \& van Mele, B. A selfhealing polymer network based on reversible covalent bonding. React. Funct. Polym. 73, 413-420 (2013).

15. Wei, Z., Lewis, D. M., Xu, Y. \& Gerecht, S. Dual cross-linked biofunctional and self-healing networks to generate user-defined modular gradient hydrogel constructs. Adv. Healthc. Mater. 6, 1700523 (2017).

16. Herbst, F., Seiffert, S. \& Binder, W. H. Dynamic supramolecular poly(isobutylene) s for self-healing materials. Polym. Chem. 3, 3084 (2012).

17. Le, H. H. et al. Triggering the self-healing properties of modified bromobutyl rubber by intrinsically electrical heating. Macromol. Mater. Eng. 302, 1600385 (2017).

18. Zhang, L., Brostowitz, N. R., Cavicchi, K. A. \& Weiss, R. A. Perspective: ionomer research and applications. Macromol. React. Eng. 8, 81-99 (2014).

19. van der Zwaag, S. (ed.). Self Healing Materials. An Alternative Approach to 20 Centuries of Materials Science. lonomers as Self Healing Polymers (Springer, Dordrecht, 2007).

20. Capek, I. Nature and properties of ionomer assemblies. II. Adv. Colloid Interface Sci. 118, 73-112 (2005).

21. Dreyfus, B. Model for the clustering of multiplets in ionomers. Macromolecules 18, 284-292 (1985).

22. Hara, M. \& Sauer, J. A. Mechanical properties of lonomers. J. Macromol. Sci, Polym. Rev. 34, 325-373 (1994).

23. Eisenberg, A. Clustering of ions in organic polymers. A theoretical approach. Macromolecules 3, 147-154 (1970).

24. Eisenberg, A., Hird, B. \& Moore, R. B. A new multiplet-cluster model for the morphology of random ionomers. Macromolecules 23, 4098-4107 (1990).

25. Kalista, S. J., Pflug, J. R. \& Varley, R. J. Effect of ionic content on ballistic selfhealing in EMAA copolymers and ionomers. Polym. Chem. 4, 4910-4926 (2013).
26. Fall, R., Master thesis "Puncture reversal of ethylene ionomers - Mechanistic Studies", Virginia Polytechnic Institute and State University, Blacksburg, VA (2001).

27. Varley, R. J. \& van der Zwaag, S. Development of a quasi-static test method to investigate the origin of self-healing in ionomers under ballistic conditions. Polym. Test. 27, 11-19 (2008).

28. Vega, J. M., Grande, A. M., van der Zwaag, S. \& Garcia, S. J. On the role of free carboxylic groups and cluster conformation on the surface scratch healing behaviour of ionomers. Eur. Polym. J. 57, 121-126 (2014).

29. Voorhaar, L. et al. Supramolecular thermoplastics and thermoplastic elastomer materials with self-healing ability based on oligomeric charged triblock copolymers. NPG Asia Mater. 9, e385 (2017).

30. Xu, C., Cao, L., Lin, B., Liang, X. \& Chen, Y. Design of self-healing supramolecular rubbers by introducing ionic cross-links into natural rubber via a controlled vulcanization. ACS Appl. Mater. Interfaces 8, 17728-17737 (2016).

31. Hohlbein, N., Shaaban, A., Bras, A. R., Pyckhout-Hintzen, W. \& Schmidt, A. M. Self-healing dynamic bond-based rubbers. Understanding the mechanisms in ionomeric elastomer model systems. Phys. Chem. Chem. Phys. 17, 21005-21017 (2015).

32. Peng, Y. et al. Strong and tough self-healing elastomers enabled by dual reversible networks formed by ionic interactions and dynamic covalent bonds. Polymer 157, 172-179 (2018).

33. Peng, $Y$. et al. Super tough and strong self-healing elastomers based on polyampholytes. J. Mater. Chem. A 6, 19066-19074 (2018).

34. Bose, R. K., Hohlbein, N., Garcia, S. J., Schmidt, A. M. \& van der Zwaag, S. Connecting supramolecular bond lifetime and network mobility for scratch healing in poly(butyl acrylate) ionomers containing sodium, zinc and cobalt. Phys. Chem. Chem. Phys. 17, 1697-1704 (2015).

35. Bose, R. K., Hohlbein, N., Garcia, S. J., Schmidt, A. M. \& van der Zwaag, S Relationship between the network dynamics, supramolecular relaxation time and healing kinetics of cobalt poly(butyl acrylate) ionomers. Polymer 69, 228-232 (2015).

36. Dahlke, J. et al. A new approach toward metal-free self-healing ionomers based on phosphate and methacrylate containing copolymers. Macromol. Chem. Phys. 218, 1700340 (2017).

37. Blackman, L. D., Gunatillake, P. A., Cass, P. \& Locock, K. E. S. An introduction to zwitterionic polymer behavior and applications in solution and at surfaces. Chem. Soc. Rev. 48, 757-770 (2019).

38. Laschewsky, A. Structures and synthesis of zwitterionic polymers. Polymers $\mathbf{6}$, 1544-1601 (2014)

39. Liu, H. et al. Zwitterionic copolymer-based and hydrogen bondingstrengthened self-healing hydrogel. RSC Adv. 5, 33083-33088 (2015).

40. Luo, F. et al. Oppositely charged polyelectrolytes form tough, self-healing, and rebuildable hydrogels. Adv. Mater. 27, 2722-2727 (2015).

41. Woodfield, P. A., Zhu, Y., Pei, Y. \& Roth, P. J. Hydrophobically modified sulfobetaine copolymers with tunable aqueous UCST through postpolymerization modification of poly(pentafluorophenyl acrylate). Macromolecules 47, 750-762 (2014).

42. Zhu, Y., Noy, J. -M., Lowe, A. B. \& Roth, P. J. The synthesis and aqueous solution properties of sulfobutylbetaine (co)polymers. Comparison of synthetic routes and tuneable upper critical solution temperatures. Polym. Chem. 6, 5705-5718 (2015).

43. Kudaibergenov, S., Jaeger, W. \& Laschewsky, A. Polymeric betaines: synthesis, characterization, and application. Adv. Polym. Sci. 201, 157-224 (2006).

44. Chen, S. et al. Development of zwitterionic polyurethanes with multi-shape memory effects and self-healing properties. J. Mater. Chem. A 3, 2924-2933 (2015).

45. Hildebrand, V., Laschewsky, A., Päch, M., Müller-Buschbaum, P. \& Papadakis, C. $M$. Effect of the zwitterion structure on the thermo-responsive behaviour of poly(sulfobetaine methacrylates). Polym. Chem. 8, 310-322 (2017).

46. Liu, P. et al. pH-sensitive zwitterionic polymer as an antimicrobial agent with effective bacterial targeting. ACS Biomater. Sci. Eng. 4, 40-46 (2018).

47. Chen, S. et al. Pyridine type zwitterionic polyurethane with both multi-shape memory effect and moisture-sensitive shape memory effect for smart biomedical application. Polym. Chem. 7, 5773-5782 (2016).

48. Guo, W., Li, X., Xu, F., Li, Y. \& Sun, J. Transparent polymeric films capable of healing millimeter-scale cuts. ACS Appl. Mater. Interfaces 10, 13073-13081 (2018).

49. Sinclair, A. et al. Self-healing zwitterionic microgels as a versatile platform for malleable cell constructs and injectable therapies. Adv. Mater. 30, 1803087 (2018). 
50. Wang, Z., Fei, G., Xia, H. \& Zuilhof, H. Dual water-healable zwitterionic polymer coatings for anti-biofouling surfaces. J. Mater. Chem. B 6, 6930-6935 (2018).

51. Ye, Z. et al. Novel dual-functional coating with underwater self-healing and anti-protein-fouling properties by combining two kinds of microcapsules and a zwitterionic copolymer. Prog. Org. Coat. 127, 211-221 (2019).

52. Perrier, S. 50th anniversary perspective: RAFT polymerization-a user guide. Macromolecules 50, 7433-7447 (2017).

53. Fleischhaker, F. et al. Glass-transition-, melting-, and decomposition temperatures of tailored polyacrylates and polymethacrylates: general trends and structure-property relationships. Macromol. Chem. Phys. 215, 1192-1200 (2014).

54. Ehrmann, M., Mathis, A., Meurer, B., Scheer, M. \& Galin, J. C. Statistical n-butyl acrylate-(sulfopropyl)ammonium betaine copolymers. 2. Structural studies. Macromolecules 25, 2253-2261 (1992).

55. Galin, M., Chapoton, A. \& Galin, J. -C. Dielectric increments, intercharge distances and conformation of quaternary ammonioalkylsulfonates and alkoxydicyanoethenolates in aqueous and tirfluoroethanol solutions. J. Chem. Soc., Perkin Trans. 2, 545-553 (1993).

56. Koeberle, P. \& Laschewsky, A. Hydrophobically modified zwitterionic polymers: synthesis, bulk properties, and miscibility with inorganic salts. Macromolecules 27, 2165-2173 (1994).

57. Low, I. M. Effects of load and time on the hardness of a viscoelastic polymer. Mater. Res. Bull. 33, 1753-1758 (1998).
58. Schneider, J. -M., Bigerelle, M. \& lost, A. Statistical analysis of the Vickers hardness. Mater. Sci. Eng. A 262, 256-263 (1999).

59. Bode, $\mathrm{S}$. et al. Correlation between scratch healing and rheological behavior for terpyridine complex based metallopolymers. J. Mater. Chem. A 3, 22145-22153 (2015)

60. Chen, S., Döhler, D. \& Binder, W. H. Rheology of hydrogen-bonded dendritic supramolecular polymer networks in the melt state. Polymer 107, 466-473 (2016).

61. den Brabander, M., Fischer, H. R. \& Garcia, S. J. (eds.). in Smart Polymers and their Applications 2nd edn. (Woodhead Publishing, 2019).

62. Bode, $\mathrm{S}$. et al. Characterization of self-healing polymers. From macroscopic healing tests to the molecular mechanism. Adv. Polym. Sci. 273, 113-142 (2016).

63. Bose, R. K. et al. Contributions of hard and soft blocks in the self-healing of metal-ligand-containing block copolymers. Eur. Polym. J. 93, 417-427 (2017).

64. Brancart, J. et al. Atomic force microscopy-based study of self-healing coatings based on reversible polymer network systems. J. Intell. Mater. Syst. Struct. $\mathbf{2 5}$ 40-46 (2014).

65. Yoon, J. A. et al. Self-healing polymer films based on thiol-disulfide exchange reactions and self-healing kinetics measured using atomic force microscopy. Macromolecules 45, 142-149 (2012).

66. Briscoe, B. J., Evans, P. D., Pellilo, E. \& Sinha, S. K. Scratching maps for polymers Wear 200, 137-147 (1996)

67. Jiang, H., Browning, R. \& Sue, H. -J. Understanding of scratch-induced damage mechanisms in polymers. Polymer 50, 4056-4065 (2009). 